\title{
Illustration of Automatic Panel Cleaning System
}

\author{
Aashika Prasad ${ }^{1}$ \\ Department of Robotics and \\ Automation, \\ PSG College of Technology, \\ Coimbatore, India.
}

\author{
Rithika $\mathrm{S}^{1}$ \\ Department of Robotics and \\ Automation, \\ PSG College of Technology, \\ Coimbatore, India.
}

\author{
Dr. Rathika PD ${ }^{2}$ \\ Assistant Professor, \\ Department of Robotics and \\ Automation, PSG College of \\ Technology, \\ Coimbatore, India.
}

\begin{abstract}
With a drive to meet the growing energy demands and to curb the increasing global greenhouse emissions, clean energy is being ubiquitously harnessed. Among these, the solar technology seems to be the pinnacle in the offing. Several research groups strive hard to attain higher efficiency in solar technology. However, one of the major causes for the drastic reduction in efficiency of the solar panels is due to the effect of soiling. Soiling takes into consideration several factors like the settling of sand particles on the solar cells, accumulation of dust coverings and bird droppings. The efficiency of Photovoltaic (PV) cells is not only limited by soiling, but also by the bird droppings on the panels and layering of the snow, which is a significant cause that has a major impact on the efficiency. Thus, to maintain efficiency throughout, the PV panels have to be cleaned and monitored regularly. Many times, the cleaning agent or the water settles behind as a layer on the panel, which again yields to soiling. Thus, there is a need to restrain the dust particles (soiling) from being settled as a layer on the panels. This paper deals with the techniques using Artificial Intelligence and Computer Vision to prevent the soiling, thereby increasing the efficiency of the solar technologies. A drone affixed with an overhead camera to monitor the panels and an end effector to carry out the cleansing process is employed. The image captured is then processed by the Machine Vision techniques to assess the type of the soiling, thereby urging the drone for removing the soiled particles. Moreover, with the addition of Artificial Intelligence and Computer Vision, we are also able to draw useful insights on the impact caused by different factors that influence soiling and these predictions would be beneficial for us in taking preventive and corrective measures, which would be an advantage in the long run.
\end{abstract}

Keywords-Automatic Panel Cleaning system, Computer Vision, Machine Learning, CNN, AI, Drone

\section{INTRODUCTION}

From the traditional solar panels on the rooftops to the floating solar farms and the solar skins, the advent of the solar panels has come a long way. The variations in the design of the panels, control of the panels, and the overall management of the panels have contributed to the increase in cell efficiency. However, the efficiency of the panels on the basis of environmental conditions is still poor. The amount of irradiance that directly reaches the solar panel determines the power output from the panel.

Researches prove that the shading of the panels is an effect of soiling. The settling of the dust particles as layers on the solar panels leads to the obstruction of the sunrays from reaching the panels. It is recorded that dust and grime have an impact of about $5 \%$ loss of performance of the panel. Cleaning these settlings naturally by means of rain is not feasible in all the regions due to the conflicting climates of the different regions.

Manual cleaning of the panels is not viable in most cases owing to the vast panel surface. Also, an erroneous or sudden movement may potentially cause scratches. Unlike the manual cleaning, autonomous cleaning of the panels will clean the surface areas of the panel equally and uniformly and can reach every nook and corner of the panels. Thereby, the use of an autonomous drone along with artificial intelligence and computer vision facilitates an increase of efficiency by inhibiting soiling.

Fig.1 illustrates the most prominent types of soiling particles observed for which the suggestions to cleaning them are provided in this paper. Removing the particles subsequently increases the efficiency of the panel.
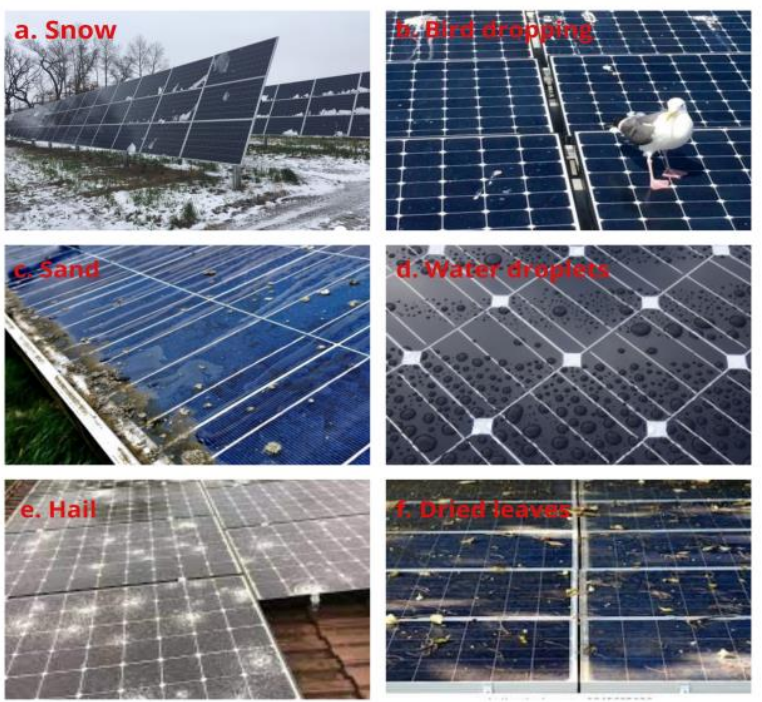

Fig.1. Different types of soiling particles affecting the panel efficiency

\section{LITERATURE SURVEY}

Of the many prevalent technologies, one of the most popular forms is the use of low volume spray nozzles that are connected to every single panel. It receives its signal from the Programmable Logic Controller [1], which also monitors and records the run time data. The set up uses a biodegradable soap concentrate that is amalgamated into the system's water line during the clean cycles. This is followed by a second rinse.

In regions where the solar panel set up is extensive, the nozzles are directly fastened to the array of the solar panels, wherein; the former is run by a microprocessor [2]. The system also has a Programmable Logic Controller and a web-based software interface, which can be scheduled and controlled by the panel operator when the need arises.

According to Kawamoto [3], a detachable cleansing system that utilizes the electro-dynamic force enables cleansing the hardly settled dust on the photovoltaic panels. The system 
is encompassed with the parallel screen electrodes that are affixed to a plastic frame. As the voltage is exerted on the parallel screen electrodes, the electro-dynamic force generated acts on the settlements on the lower electrode and produces a flip-flop motion which passes to the upper screen electrode and the settlements from the panel is unfastened and slides down along the panel due to the gravitational force of the earth. This practice gives higher efficiency and can be implanted for the panels that are devised in the deserts.

According to Huang [4], the use of the fuzzy logic technique yields an increase in efficiency prompting in intelligent solar panel cleaning system. The light sensor is positioned parallel to the direction of the sunlight. The data collected from the light sensors are incorporated with the fuzzy logic control software after which the stepper motors control the cleaning process. This is carried on for a definite period, thereby maintaining efficiency. In addition to this, a nozzle spray is triggered on, which limits the temperature of the solar cells also adding to the efficiency of the panel.

Park and Im [5] stated that the use of the superhydrophobic and water-repellent surface on the PV panels has enhanced the efficiency of the panels along with a micro-shell array put up on a flexible and transparent Polydimethylsiloxane elastomer surface. The PDMS adds to its trait of waterrepellence and has a superior effect on dust cleaning. The micro shell PDMS had a better and a greater effect on the cleansing of the panels than the flat PDMS. The experiment proved that sheathing the solar cell module with a super-hydrophobic array of micro-shell PDMS could reduce soiling.

A conventional approach to the automatic panel cleaning is that the system comprises a reservoir that stores the soap concentrate, a sediment filter that holds water softener media, and an anti-siphon valve that obstructs the backwashing into the system. [6] The nozzles are attached to the PV modules. The controller is mechanized to control the wash and the rinse cycles, which can be modified periodically.

\section{EFFICIENCY COMPUTATION}

The parameter of the solar cell has to be monitored regularly to identify the cause for the drop in efficiency. The efficiency will be continuously monitored in real-time for each of the panel modules and the values are passed on to the computer system where the entire solar cells are being controlled by a centralized system. The environmental conditions are not always favorable and thus the average of a particular duration's data is taken into consideration.

The efficiency of the cells can be computed by finding the ratio between the output energy from the solar cell to the input energy from the sunlight. Hence efficiency must be tracked carefully and precisely to analyze the performance of the solar cell.

The intensity of the solar radiation does not remain the same throughout the year. It keeps varying constantly and is highly dependent on the climatic conditions. For instance, the intensity of the radiation is maximum during the summer and is relatively lower in the winter. This trend changes with respect to the geographic locations as well. Thereby, keeping this in mind, to improve and maintain the efficiency of the panels, the efficiency threshold is preset in accordance to the changes in the climate.

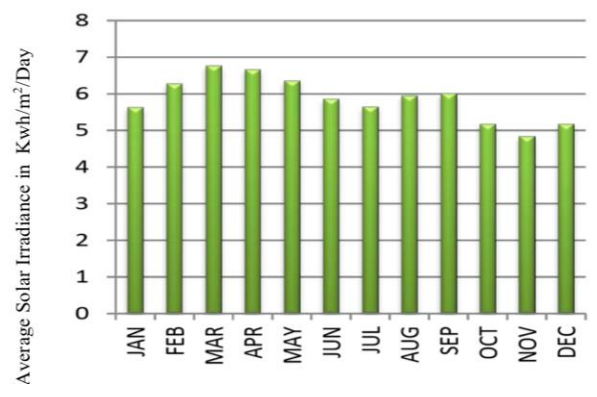

Fig.2. Average solar irradiance falling on the panel [11]

The efficiency of the solar cell is given by,

Where,

$$
\eta=\frac{\text { Voc Isc } F F}{\operatorname{Pin}}
$$

$\mathrm{V}_{\mathrm{oc}}$ is the open-circuit voltage;

$\mathrm{I}_{\mathrm{sc}}$ is the short-circuit current;

FF is the fill factor and

$\eta$ is the efficiency.

A threshold value is set initially and when the efficiency of a specific cell drops below the threshold value, the control techniques are performed automatically. Also, the efficiency of one cell is compared with others to check the abnormalities. Settling of various particles leads to a continuous drop in efficiency. When left unnoticed, these particles tend to accumulate leading to a huge drop in efficiency throughout. Each particle affects the efficiency in a unique way. The drop in efficiency depends on the mass, texture, color and similar factors of every particle. Fig. 3 depicts that the output power reduces in accordance to the particle settled on the panel. Fig.4 shows the loss of efficiency due to the dust settled on the panels.

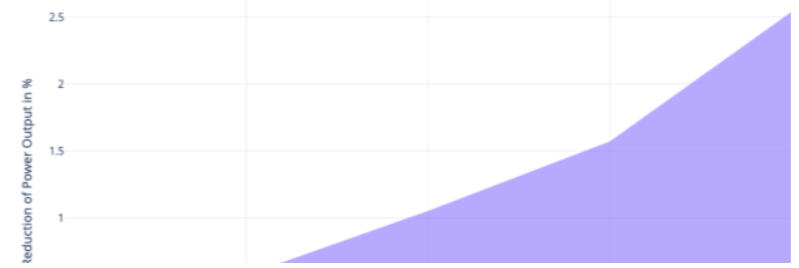

Fig.3 Reduction of output power in terms of \% due to soiling effects [12]

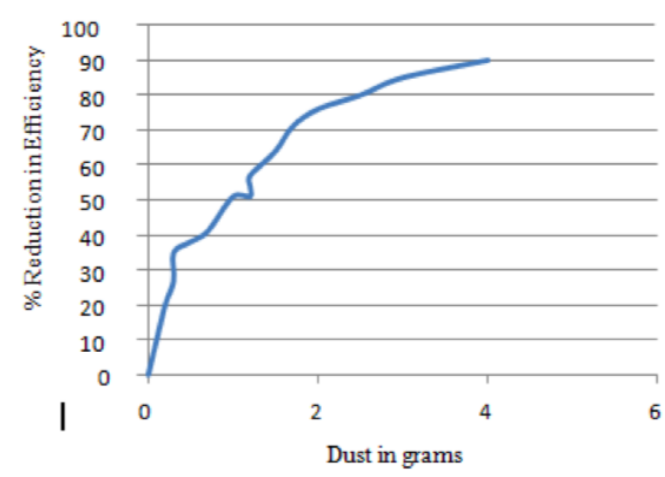

Fig.4. Reduction in the efficiency of the panels due to dust [12] 


\section{METHODOLOGY}

The efficiency of each solar panel is being computed continuously with respect to its output voltage and current, and is fed to a centralized system. The average of the data of a particular duration is taken into consideration as efficiency is directly influenced by the climatic conditions. If the efficiency drops below a certain threshold, the drone is actuated by the ground subsystem to carry out its operations. A ground subsystem has a Human Machine Interface (HMI) panel that displays the abnormalities, battery indication, and so the operator can control the operations if required and in case of emergencies. The camera affixed over the drone captures the image of the cell that lacks efficiency and through various vision techniques, analysis is being carried out. The coordinates where the abnormalities are found are detected using these techniques and controlling techniques are performed over these using the end effectors on the drone.

Also, this analysis using vision technique is used alongside with Artificial Intelligence to make the process more effective and useful in the long run. The data set that is stored in the ground subsystem is to be classified to acquire the various patterns from the image. This can be done using the CNN algorithm as the Convoluted Neural Networks produce results that are highly efficient, reliable and faster.

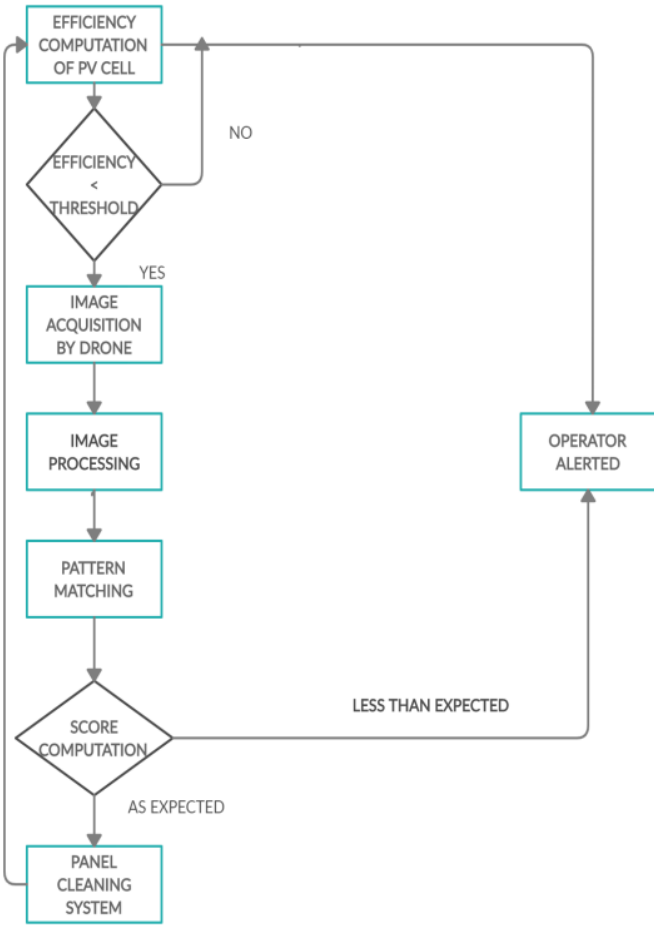

Fig.5. Block Diagram of the proposed model

\section{DRONE TECHNOLOGY}

The drone is encompassed with two subsystems - an on-board subsystem and a ground subsystem. The on-board subsystem comprises of the vision system with the techniques of image processing embedded on it. It is also incorporated with flight control software and a communication interface that interfaces the flight control with the ground subsystem. On the other hand, the ground subsystem comprises a computer, thereby enabling an efficient interaction with the on-board system. It also comprises a communication interface and a data storage unit in order to facilitate the interaction with the onboard system and thereby storing the data in the latter.

A thermal camera is affixed on the drone, which highly aids in detecting the hotspots, cracks, and the temporary shadowing defects such as dust and bird droppings on the panel (Fig 1). The difference in the temperature between the cells of the panels is so accurate that it enables in observing the defects at an early stage. The connection between the thermal camera and the ground subsystem provides the coordinates of defects on the panel after which the panels can be cleansed. Also, the status of the battery life is indicated on the HMI present on the ground subsystem, and when it drops the operator is alerted.

The end effectors that are fastened to the drone are blowers to blow the dust away, vacuum suction to remove the bird droppings, and brushes with sponge to scrub all the other soiled particles. The wire bristles are soft, very easy to clean and is also resistant to heat. A spray tank is attached on top of the drone and is controlled by a spray block control system. Fig.6 and Fig.7 represents the Solidworks model of the drone capturing the data and cleaning the panel.

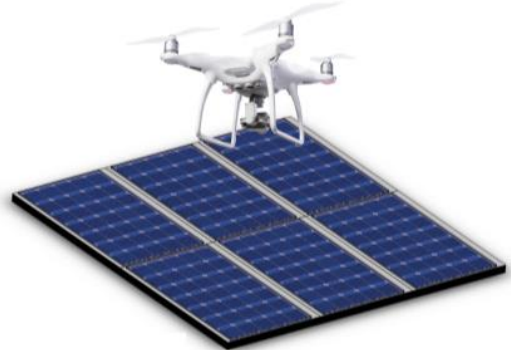

Fig.6. Solidworks model of a vision and data capturing drone

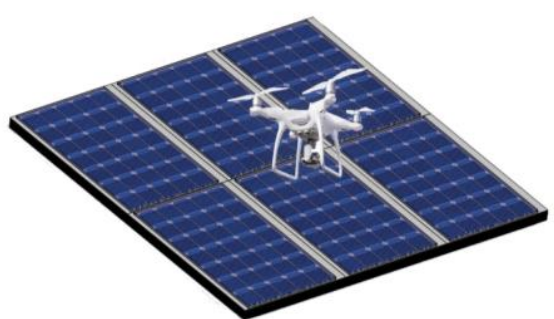

Fig.7 Solidworks model of the drone cleaning the panel

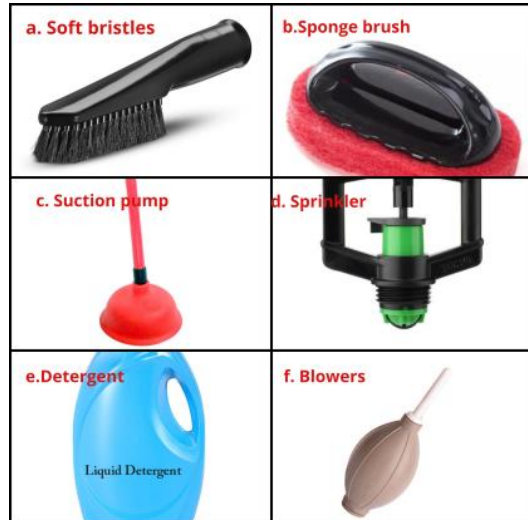

Fig.8. Different types of end-effectors employed 
The drone for the recommended purpose is expected to be highly resistant to various external factors which includes exposure to the ultra-violet rays, humidity, rain and heat and other similar conditions because the drone works completely in the rough and harsh external environment. DJI offers a wide range of solutions for operating drones to necessitate the cleaning of the solar panels. The features that has to be considered significantly for choosing a drone includes the right selection of the payload, the wind resistance offered by the drone, its operating temperature and its maximum transmitting distance. The payload has to be adopted in such a way that it is sufficient to hold the weight of the camera and the end effectors as well. The transmitting distance has to be chosen depending on the area to be covered by the drone. It has to be high if the drone has to cover a large area and low in case of implementing it over a range of few kilometers. The battery capacity of the drone can be compromised as the drone is required to perform the cleaning action only when there is a drop in efficiency. Thereby, considering the following aspects, the DJI MATRICE 200 series is one of the good options where the additional cameras and end effectors can be attached to these readily available drones. Apart from this Vulcan UAV Raven, Onyxstar Hydra and Tarot T series are some of the other readily available drones that can be used after attaching the cameras and the end effector to it.

\section{VISION TECHNIQUES}

The drone that flies over the solar panels captures the image of the solar panels and its cells through the overhead camera that is mounted on the drone. As the efficiency of the solar cells drops below the minimum value of the threshold, the drone gets actuated and advances to the particular cell. It reads the image captured by the overhead camera and processes it using the techniques of Computer Vision.

The process involves considering a pattern-matching algorithm for processing the image. Pattern matching rapidly pinpoints the regions of a grayscale image that matches a known reference pattern, which is depicted as a reference model or template. A template for the model is devised such that the required output model is matched with the reference model. Considering this, the image of the solar cells on the panel is captured initially during the setup of the panels, and this image is used as a template for pattern matching. The Machine Vision application then looks for instances of the reference model in every image acquired and thereby generates a score for each match. This score relates to how closely the template resembles the located matches. The pattern matching finds and detects the template matches regardless of the variation in lighting, blur, noise, or any geometric transformation. If the score in the pattern matching drops below the pre-set threshold value, the coordinates are sent to the system for further analysis through the sensor.

The image of the sand particles, dust accumulation, cracks, and bird droppings are captured by the camera and are BGR image. This is converted into a grayscale image so as to reduce the complexity of the image that is in BGR thereby making it relatively facile to deal with a single-color channel rather than a multi-color channel. Following this, the image is applied with the Gaussian Blur feature, which smoothens the image thereby minimizing the noise level and the speckles present in the image.

Subsequently, the edge detection technique is applied to the image. This is performed to detect the cracks on the solar cells. Cracks on the panels can lead to a large drop in the efficiency of the working of the solar panels. Hence, it is indispensable to keep an eye on the very minute of the cracks on the panel. Using this edge detection helps to find edges and boundaries along a line of pixels in the image. This comes off by finding the discontinuities in the brightness. When the cracks are detected in edge detection, the coordinates of the abrupt change are sent to the system for repair or replacement of the cell.

Trailing this is the contour detection that not only helps in edge detection but also determines the shape of the objects like sand coverings, dust particles, and bird droppings. The output at this stage is used for the purpose of pattern matching using CBIR. CBIR compares and matches the pattern from the output with the reference pattern. Content Based Image Retrieval is the approach where the search for a pattern based on the content of the query image as in color, texture, and shape are made. As soon as the pattern is matched with the reference, the drone is given a command to clean the particular coordinate of the panel. Fig. 9 gives the block diagram designed for the system.

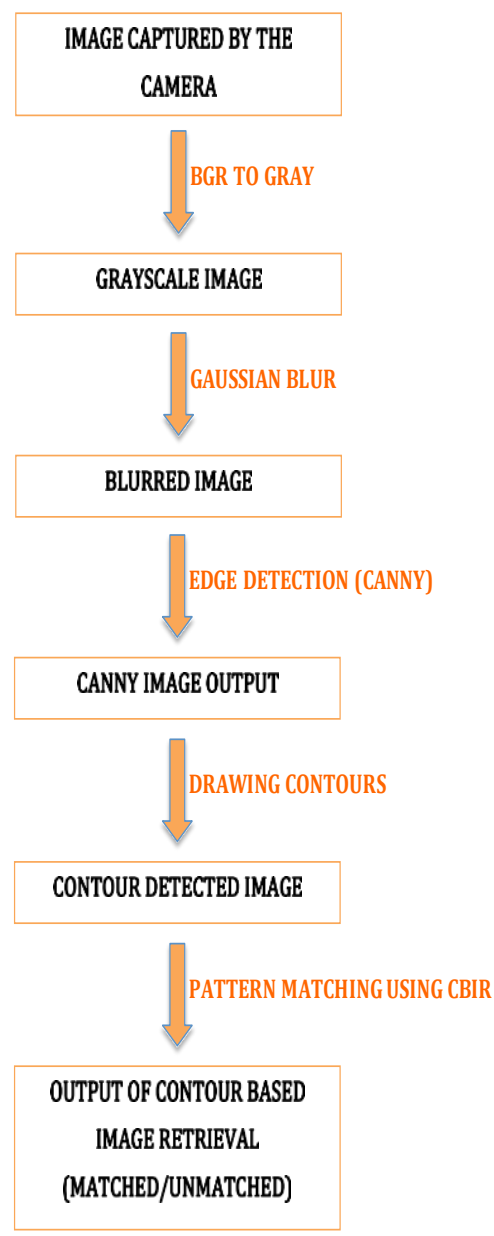

Fig.9. Block Diagram of the Image Processing Techniques 


\section{ARTIFICIAL INTELLIGENCE}

Artificial Intelligence is a technology, which has already influenced most of the technological fields, and deep learning and machine learning are the foremost parts of Artificial Intelligence. This is a flourishing field in the present era as it can make systems understand data by itself with very little human intervention and various analyses can be performed.

Application of Convolutional Neural Network aids us by producing more efficient and faster results. As the network trains on the images, it automatically extracts the features from it. It takes the input image and applies a filter on it, thereby creating a feature map. After several such feature maps being employed to the image, an image of the convoluted layer is obtained. The weights and the features are trained using the forward and the backward propagation training after which the patterns are classified respectively.

Applying CNN to the processed image does the selection of the end effector for the cleaning process. The network reads the input images and gives the proportionate end effector for the proportionate dirt. The adoptions of the end effectors are chosen in such a way that it is a combination of two or more end effectors appropriate for the soiled particle. For instance, if the particle is a bird dropping, a combination of sprinkler and the sponge brush as the end effector can be chosen for a particular period of time.

The input images typify the various soiled particles. The number of input nodes with images of a particular pixel size is chosen in accordance with the number of input images, say for six input images, six input nodes are chosen, thereby on applying the filter, the image gets convoluted. Following this technique, the image undergoes pooling, which takes in the large images and thereby shrinks them down while upholding the most salient details in them. This condenses the image to a quarter of the input image, that is, the image has lesser pixels. This is further carried with the ReLU activation, which returns the image of the same size as that of the pooling, but with all the negative values eliminated from it, thereby producing dense and fully connected layers. The output layer will have six nodes, one for each end effector with softmax activation.

\section{RESULTS}

The degradation of the output energy and the reduced efficiency of the solar panels caused by the temporary shadowing defects (soiling) can be overcome by the use of the proposed system. The system is self-sustainable throughout the varying weather conditions and climatic conditions. The drone can scour the soiled particles obtained from the continuous monitoring of real-time data. The drone can hover around the entire stretch of the panel and can operate ceaselessly, thereby sustaining a very good efficiency throughout.

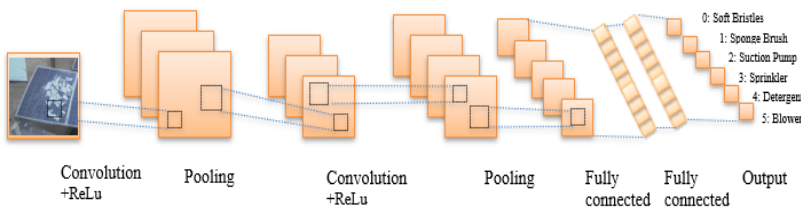

Fig.10. Block Diagram of the CNN Implementation
The following images are obtained after the image has undergone the mentioned image processing techniques. (Fig.11 to Fig. 15)

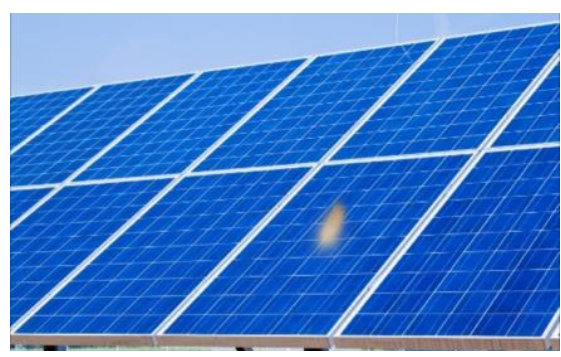

Fig.11. Image captured by the drone

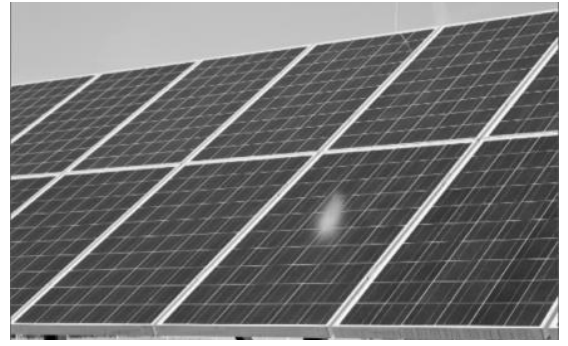

Fig.12. Captured image converted to grayscale

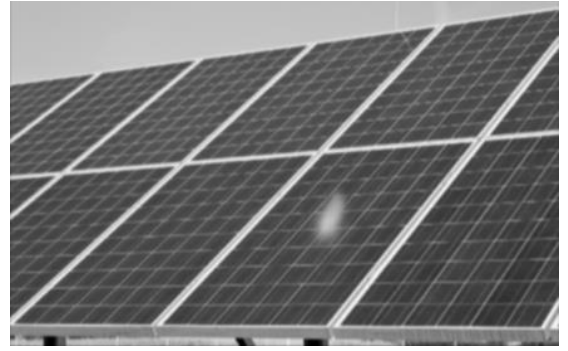

Fig.13. Image applied with Gaussian Filter

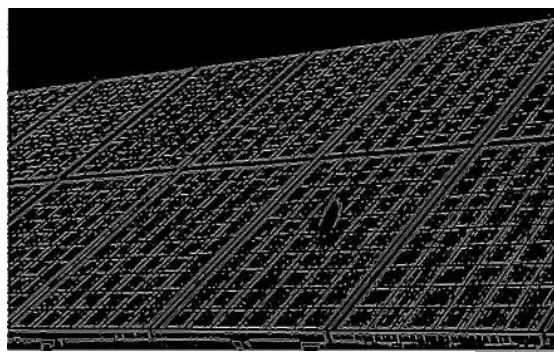

Fig.14. Edge Detection using Canny

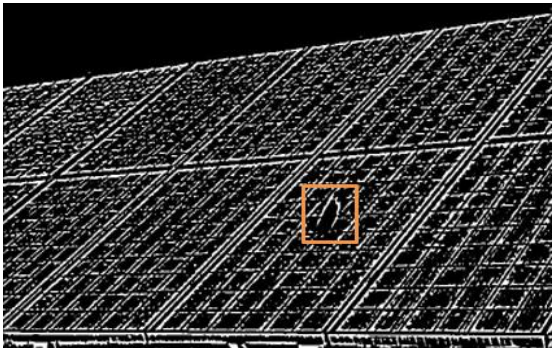

Fig.15. Contour detected image 


\section{CONCLUSION AND FUTURE WORKS}

The investment in solar panels is already vastly altered and will continue to grow so in the future. Maintaining the efficiency of the panels is an obligation. Thereby utilizing the principles of Machine Vision and Artificial Intelligence, this solar panel cleaning system ensures to maintain the solar panels throughout, which is otherwise a laborious process. This system is certain to increase the overall efficiency of the PV system.

The installations of solar panels have massively grown over the last few years. Hence it is indispensable to use this resource effectively by maintaining a high efficiency. Adopting the autonomous drones for the inspection and cleaning of the PV cells can cut down labor costs and long labor hours in hazardous conditions. Also, regardless of what the environmental conditions are, the proposed system can work credibly, resulting in a higher accuracy. Subsequently, the effectiveness of the inspection is greatly increased by the quality and the quantity of the data acquired. This technology can be implemented irrespective of the conditions, ranging from a small-scale roof panels to large solar farms.

Our future works include selection of a large dataset for training the model and thereby obtaining the accurate predictions for every input sample. It also includes implementing the proposed work as a real time system and also focusing to choose features such that the efficiency of the panels is much higher than the existing technological systems.

\section{REFERENCES}

[1] Gargi Ashtaputre, Amol Bhoi, "Artificial intelligence based solar pane cleaning robot,", IOSR Journal of Electronics and Communication Engineering (IOSR-JECE) e-ISSN: 2278-2834,p- ISSN: 2278-8735.Vol 14, Issue 3, Ser. II (May-June. 2019), PP 12-15.

[2] Tatiani Pivem, Felipe de Oliveira de Araujo, Laura de Oliveira de Araujo, Gustavo Spontoni de Oliveira, "Application of a computer vision method for soiling recognition in photovoltaic modules for autonomous cleaning robots," Signal \& Image Processing: An International Journal(SIPIJ) vol.10, No.3, June 2019

[3] Swanand S. Wable, Somashekhar Ganiger, "Design \&amp; manufacturing of solar panels cleaning system,'International Journal for Research in Applied Science \& amp; Engineering Technology(IJRASET), vol.5, July 2017
[4] Al-Dahidi, Sameer \& Ayadi, Osama \& Adeeb, Jehad \& Alrbai, Mohammad \& Qawasmeh, Bashar. (2018), "Extreme learning machines for solar photovoltaic power predictions," Energies. 11. 2725 . 10.3390/en11102725. 11(10), 2725; https://doi.org/10.3390/en11102725, Received: 3 September 2018 / Revised: 7 October 2018 / Accepted: 9 October 2018 / Published: 11 October 2018.

[5] Amy J.C. Trappey, Paul P.J Chen, Charles V. Trappey, Lin Ma, “A machine learning approach for solar power technology review and patent evolution analysis,"Applied Sciences,2019.

[6] Grando, Marcel \& Maletz, Elias \& Martins, Daniel \& Simas, Henrique \& Simoni, Roberto, "Robots for cleaning photovoltaic panels," State of the Art and Future Prospects, 2017.

[7] Kavya V, Raam M. R. Keshav, "Solar dust detection system," International Conference on Power Energy, Environment and Intelligent Control (PEEIC) ,pp.138-140, 2018.

[8] Gaofa He, Chuande Zhou, Zelun Li, "Review of self-cleaning method for solar cell array",International Workshop on Automobile, Power and Energy Engineering, 2011

[9] Kawamoto H, Shibata T, "Electrostatic cleaning system for removal of sand from solar panels," Journal of Electrostatics, Vol 73,pp.65-70,Feb 1,2015

[10] Ahmed, Zeki A, Hussein A Kazem,Kamaruzzaman Sopian, "Effect of dust on photovoltaic performance," Review and Research Status, 2013.

[11] Ananthi K, Priyadharshini R,Arshad N, David E, "Increasing the efficiency of solar panels using autonomous cleaning robot,"International Journal of Pure and Applied Mathematics, Vol 119, pp.837-847, 2018.

[12] Sulaiman, Shaharin Anwar, Fiseha M. Guangul, Mohamad Nur Hidayat Mat and Mohammed A Bou-Rabee, "Real-time study on the effect of dust accumulation on performance of solar PV panels in Malaysia," International Conference on Electrical and Information Technologies (ICEIT) pp.269-274, 2015.

[13] Campoy, Pascual \& Correa, Juan Fernando \& Mondragón, Iván \& Martinez, Carol \& Olivares-Mendez, Miguel \& Mejias, Luis \& Artieda, Jorge. (2009). Computer vision onboard UAVs for Civilian tasks. Journal of Intelligent and Robotic Systems. 54. 105-135. 10.1007/s10846-0089256-Z.

[14] Pivem, Tatiani \& Araujo, Felipe \& Araujo, Laura \& Oliveira, Gustavo. (2019). Application of A computer vision method for soiling recognition in photovoltaic modules for Autonomous cleaning Robots. Signal \& image processing : An International Journal. 10. 43-59. 10.5121/sipij.2019.10305.

[15] Al-Dahidi, S.; Ayadi, O.; Adeeb, J.; Alrbai, M.; Qawasmeh, B.R. Extreme learning machines for solar photovoltaic power predictions. Energies $2018,11,2725$

[16] Trappey, Amy \& Chen, Paul \& Trappey, Charles \& Ma, Lin. (2019). A Machine learning approach for solar power technology review and paten evolution analysis. Applied Sciences. 9. 1478. 10.3390/app9071478. 